\title{
Microstructure of a Cu film grown on bcc Ta (100) by large-scale molecular-dynamics simulations
}

\author{
Ivan Lazić, ${ }^{1,2, *}$ Peter Klaver, ${ }^{1}$ and Barend Thijsse ${ }^{1}$ \\ ${ }^{1}$ Department of Materials Science and Engineering, Faculty of 3mE, TU Delft, Mekelweg 2, $2628 \mathrm{CD}$ Delft, The Netherlands \\ ${ }^{2}$ Faculty of Applied Sciences, Kavli Institute of Nanoscience, TU Delft, Lorentzweg 1, 2628 CJ Delft, The Netherlands
}

(Received 7 August 2009; revised manuscript received 2 October 2009; published 13 January 2010)

\begin{abstract}
Molecular-dynamics simulations using embedded atom method potentials were carried out to study the growth and subsequent annealing of a 6.3 monolayer thick $\mathrm{Cu}$ film on a bcc-Ta (100) substrate of a very large area $\left(100 \times 100 \mathrm{~nm}^{2}\right)$. The purpose is to obtain, for this typical example of a crystallographically incompatible system, atomic-level insight into the microstructural evolution of the film/substrate interface and the film itself, with the limiting influence of in-plane boundary conditions kept to a minimum. It is found that the first $\mathrm{Cu}$ plane grows heteroepitaxially on the bcc-Ta (100) surface. The second $\mathrm{Cu}$ plane is the most interesting plane; it grows in the form of a pattern of 26-atom misfit supercells which after prolonged film deposition relax energetically, by breaking up in groups and forming $30 \AA$ wide in-plane island strips separated by fcc $(1 / 6)\langle 211\rangle$ vectors. The distorted monolayer represents a way of enabling epitaxy between very different crystal structures without introducing misfit dislocations. The third and higher $\mathrm{Cu}$ planes are fcc (111) planes. The $\mathrm{Cu}$ film is polycrystalline, starting from the second plane up, with two different in-plane crystal orientations. The influence of a one-monolayer Ta terrace on the substrate is minimal. Upon annealing the film at elevated temperatures, a tendency toward island coarsening and agglomeration is observed, but a full threedimensional island formation such as found experimentally is not observed. Compared to an earlier simulation on a smaller substrate area $\left(18 \times 18 \mathrm{~nm}^{2}\right)$, the current simulations have produced a much richer microstructure. The present results therefore warn against the use of too small simulation domains when complex strain fields can be expected.
\end{abstract}

DOI: 10.1103/PhysRevB.81.045410

PACS number(s): 68.55.-a, 81.15.-z, 07.05.Tp, 02.70.Ns

\section{INTRODUCTION}

Understanding the growth of $\mathrm{Cu}$ thin films on Ta substrates is fundamentally as well as technologically important. The fundamental importance is due to the fact that $\mathrm{Cu}-\mathrm{Ta}$ is a prime example of a strongly heterogeneous film-substrate system: in equilibrium $\mathrm{Cu}$ has the fcc and Ta has the bcc crystal structure, the atomic diameters differ by a factor of 1.12 , the bulk and shear moduli both differ by a factor of 1.43 , the cohesion energies differ by a factor of 2.29 , and the melting temperatures differ by a factor of 2.92 , with in all cases Ta having the greater value. The technological importance lies in modern integrated circuit (IC) interconnects. Tantalum is a diffusion barrier material, and the microstructure of the $\mathrm{Cu}$ film affects its adhesion to Ta and the microstructure of the subsequent $\mathrm{Cu}$ fill (electromigration resistance). Atomistic simulations can provide new insights in the formation and structure of $\mathrm{Cu}$ films grown on Ta substrates.

In earlier work, classical molecular-dynamics (MD) results of $\mathrm{Cu}$ deposition on bcc-Ta (100) and (110) (Ref. 1) and $\beta$-Ta (001) (Ref. 2) were reported ( $\beta$-Ta is a metastable form of Ta that is sometimes found in thin films). While much new information was obtained from these simulations, the Ta substrates were only modest in size $\left(18 \times 18 \mathrm{~nm}^{2}\right)$. Therefore, the in-plane periodic boundary conditions may very well have altered certain growth phenomena in the film, especially those involving extended stress fields. For instance, it was observed that the $\mathrm{Cu}$ film growing on the bcc-Ta (100) was monocrystalline from the third layer onward. This might be an indication that the system was too small, at least so small that different in-plane grain orientations-and therefore grain boundaries (GBs) - were artificially suppressed. It was therefore decided to continue the work and simulate a much larger system. With boundary condition effects reduced by an order of magnitude and with step edges of various orientations added to the substrate, it was expected that a richer and more realistic variety of crystal defects could be studied.

In this work we report simulations of $\mathrm{Cu}$ growth on a bcc-Ta (100) substrate of an unusually large size, 100 $\times 100 \mathrm{~nm}^{2}$. Prior to $\mathrm{Cu}$ growth, a single-monolayer (ML) Ta terrace was added on top of the substrate in order to be able to study possible step-edge effects in the same simulation run. We will discuss the host of phenomena that emerged in the successive atomic $\mathrm{Cu}$ planes during and after the deposition: heteroepitaxy, grain boundaries, dislocations, stepedge effects, point defects, and texture. It will be shown that, after a few initial planes exhibiting different types of morphology, a textured polycrystalline fcc-Cu (111) film develops. The analysis of the symmetry types of the local atomic angular environments turned out to be a very successful tool in this study, and we will explain how this is done. Very recently, atomistic simulations of surface wetting and dewetting in the $\mathrm{Cu}$-Ta system were reported by Hashibon et al. ${ }^{3} \mathrm{~A}$ number of differences with the current work make it difficult to compare the results. Most notably, Hashibon et al. placed a $\mathrm{Cu}$ film against a Ta substrate, rather than performing an actual deposition simulation, which is a rather unphysical approach and would likely result in a film that would dewet more easily than one that was deposited atom by atom. Also, their Ta substrate was (110) oriented and much smaller in size, approximately 40 times smaller in area, and they used another potential than ours. In another recent work, ${ }^{4} \mathrm{Cu}$ (111) and $\mathrm{Nb}(110)$ crystal slabs were brought together in the Kurdjumov-Sachs ${ }^{5}$ orientation relation, and the $\mathrm{Cu}$ (111) 
plane closest to the interface was homogeneously strained in a well-argued effort to design a plane that lowers the interface energy. Because of the very close similarity of the $\mathrm{Nb}$ and Ta lattice constants $(0.03 \%$ difference), this work effectively functions as a verification "by design" what our earlier work on $\mathrm{Cu}$ (111) growth on $\mathrm{Ta}$ (110) has shown "by simulation." Although in both cases the Kurdjumov-Sachs orientation between the bcc and fcc crystals was found, in agreement with the experiment, the designed and strained $\mathrm{Cu}$ (111) interface plane had only $0.7 \%$ fewer atoms than the subsequent free $\mathrm{Cu}$ (111) planes, whereas the simulations yielded an interface plane containing 3.2\% fewer atoms, with the lower number being due to complicated superstructures which by themselves also contain irregularities. Such examples show that simulations in some cases can yield physically realistic results that go beyond theoretical predictions. In addition, the present paper deals with $\mathrm{Cu}$ on $\mathrm{Ta}$ (100) rather than $\mathrm{Ta}$ (110). An approach of designing the first $\mathrm{Cu}$ interface plane similarly to the one just described ${ }^{4}$ would certainly fail, because we will show that it is the second $\mathrm{Cu}$ plane that functions as the interface plane and that this plane contains defects and superstructures that would be very hard to predict in a design effort.

\section{COMPUTATIONAL}

The MD simulations were performed on four dual core computers using the parallelized version of the Delft molecular-dynamics code CAMELION and applying Johnson-Oh embedded atom method (EAM) potentials for $\mathrm{Cu}$ (Refs. 6 and 7 ) and $\mathrm{Ta}^{8}$ The $\mathrm{Cu}-\mathrm{Cu}$ interaction has a cutoff radius of $4.83 \AA$, which includes the equilibrium first-, second-, and third-nearest-neighbor distances (2.56, 3.61, and $4.43 \AA$ ). The Ta-Ta interaction has a cutoff radius of $3.99 \AA$, which includes the equilibrium first- and secondnearest-neighbor distances $(2.86$ and $3.30 \AA)$. For the $\mathrm{Cu}-\mathrm{Ta}$ cross potential we have used the unlike-atom pair potential form by Johnson, ${ }^{9}$ in a slightly modified form. This potential was fitted to the (positive) heat of formation of a B2 (CsCltype) $\mathrm{Ta}-\mathrm{Cu}$ alloy, $0.03 \mathrm{eV} /$ atom. This value was determined according to the Miedema model, ${ }^{10}$ although more recent $a b$ initio calculations show that the actual value is probably $\sim 0.1 \mathrm{eV} /$ atom higher. Nevertheless, in either case the interface will behave energetically as a rather inert region. Also, although the potential contains no explicit angular interaction terms, it was found that it describes the $\beta$ phase of Ta, which has a rather complex structure, quite well. ${ }^{11,12}$ Further details of the potential are given in Ref. 13 .

In the MD simulations, the $\mathrm{Cu}$ atoms were deposited onto a 7 ML thick $100 \times 100 \mathrm{~nm}^{2}$ bcc-Ta (100) Ta substrate, with in-plane periodic boundary conditions. The in-plane number of unit cells was $303 \times 303=91809$. On the substrate a wing-shaped single-monolayer Ta terrace was added, covering $44.7 \%$ of the surface [Fig. 1(a)]. In total the substrate contained 683678 atoms. The volume and temperature were kept constant in the simulation. The incident deposition angle of the $\mathrm{Cu}$ atoms was $15^{\circ}$ off normal, the same angle as experimentally used in $\mathrm{Cu}$ film growth on $\mathrm{Ta}$ for thermal helium desorption experiments. ${ }^{14}$ The azimuthal incident di-

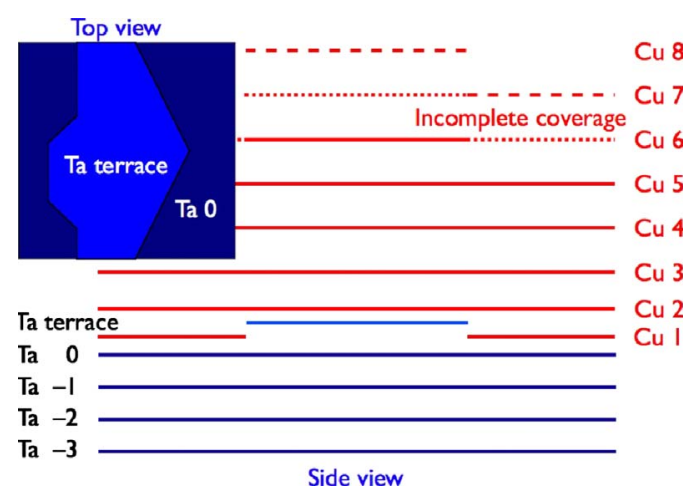

(a)

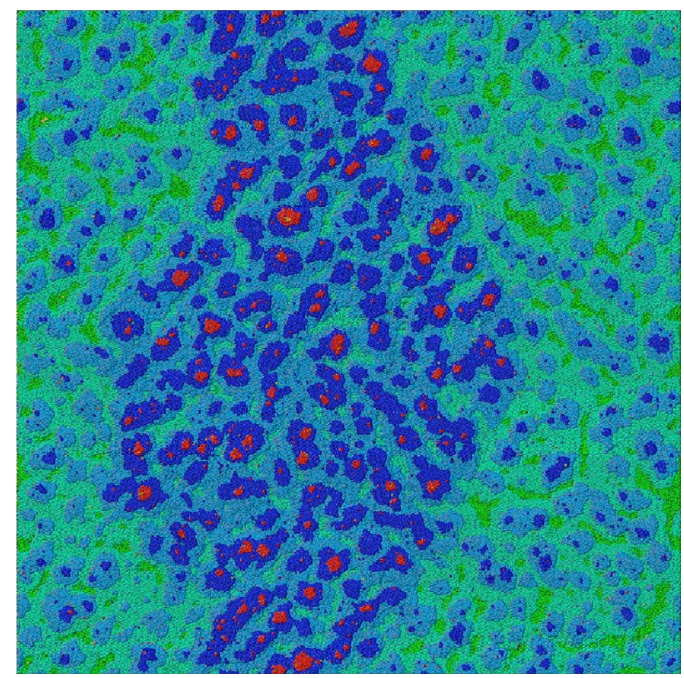

(b)

FIG. 1. (Color online) (a) Schematic side and top views of the bcc-Ta (100) substrate with terrace and the first eight $\mathrm{Cu}$ planes. Plane numbers as used in this work are indicated. In reality the substrate contains three more Ta planes. (b) Top view of the final $\mathrm{Cu}$ film, after 6.3 ML deposition; atoms colored according to height. The red atoms are in plane $\mathrm{Cu} 9$.

rection of each $\mathrm{Cu}$ atom was chosen randomly, to avoid a specific relation to the crystal structure of the substrate, as was the initial position of each atom in the top of the simulation box. The atoms arrived with a kinetic energy of 0.17 $\mathrm{eV}$, a typical magnitude for electron-beam evaporated atoms. In total $1016035 \mathrm{Cu}$ atoms were deposited. As we will see, the first deposited monolayer of $\mathrm{Cu}$ can be counted as 92000 atoms and all subsequent monolayers as 174000 atoms. Both numbers are approximate. The full deposition run has produced a 6.3-ML-thick $\mathrm{Cu}$ film, covering a vertical range of more than seven planes [Figs. 1(a) and 1(b)].

In order to finish the simulation in a feasible amount of time, the deposition rate in the simulation was taken as $1 \AA / n s$, which is nine orders of magnitude faster than in typical experimental conditions $(1 \AA / s)$. In order to permit the system nevertheless a realistic opportunity for thermal relaxation and surface diffusion in the simulations, we have adopted the elementary method of giving the film and substrate atoms a higher mean kinetic energy in the simulations than in the experiments $(300 \mathrm{~K})$. To compensate the deposi- 
tion rate fully (at least, for as far as time and temperature can be considered exchangeable), a deposition temperature of $1000 \mathrm{~K}$ would be necessary. This can be seen from the simple transition-state-theory estimate $N=v_{0} t \exp (-Q / k T)$ for the number of jumps $N$ of activation energy $Q$ that an atom will execute in time $t$ at temperature $T$. Taking $v_{0}$ $=10^{13} \mathrm{~s}^{-1}$ and $t$ equal to, say, half the monolayer deposition time in the experiment $(1 \mathrm{~s}$ at $300 \mathrm{~K})$, an atom will execute jumps with $Q<0.78 \mathrm{eV}$ at last once in the experiment $\left(N_{\exp }>1\right)$. In the simulations, where $t=1 \mathrm{~ns}$, one would need $T=1000 \mathrm{~K}$ to reach the same situation $\left(N_{\text {sim }}>1\right)$. Note, however, that such a $1000 \mathrm{~K}$ simulation would still reproduce a $300 \mathrm{~K}$ experiment with a systematic distortion of the kinetics. For example, the ratio $N_{\text {sim }} / N_{\text {exp }}$ is less than 1 for all $Q$ smaller that $0.78 \mathrm{eV}$ and larger than 1 for all $Q$ bigger than $0.78 \mathrm{eV}$, which means that the relative rates of microscopic events essential for relaxation will very likely be affected. More importantly, the simulation temperature should not be too close to the melting point of the simulated materials, or else undercooled liquids (glasses) may form. Since $\mathrm{Cu}$ has a melting temperature of $1356 \mathrm{~K}$ (1365 K with the Oh-Johnson potential used), such problems have indeed been observed in trial deposition simulations at $1000 \mathrm{~K}$. As it was found that all dubious high-temperature effects had disappeared from the simulations at $750 \mathrm{~K}$, it was decided to run the full simulations with the film-substrate system thermostat set at $750 \mathrm{~K}$. The exact effects of the limited $\mathrm{Cu}$ mobility compared to a $1000 \mathrm{~K}$ simulation are hard to estimate since on a growing surface many different activation energies are in operation. Yet, in order to give an impression of the kinetic downscaling from 1000 to $750 \mathrm{~K}$, we mention that in a simulation of $\mathrm{Cu}$ deposition onto $\mathrm{Cu}$ at $1000 \mathrm{~K}$, the $\mathrm{Cu}$ atoms move up to $\sim 8 \mathrm{~nm}$ away from the positions where they are adsorbed. At $750 \mathrm{~K}$, this distance is only $\sim 3 \mathrm{~nm}$.

Finally, carrying out the simulation at $750 \mathrm{~K}$ instead of $300 \mathrm{~K}$ with unmodified potentials would create unphysical thermal stresses between $\mathrm{Ta}$ and $\mathrm{Cu}$ since the thermal expansion coefficient of fcc $\mathrm{Cu}\left(16.5 \times 10^{-6} / \mathrm{K}\right)$ is 2.6 times larger than that of bcc $\mathrm{Ta}\left(6.3 \times 10^{-6} / \mathrm{K}\right)$. Therefore, the $\mathrm{Cu}-\mathrm{Cu}$ potential was slightly adapted to make the equilibrium lattice spacing at $750 \mathrm{~K}$ equal to the experimental value at $300 \mathrm{~K}$. We did not change the Ta potential because of its substantially lower expansion coefficient.

\section{LOCAL SYMMETRY TYPE}

In order to attribute a local crystal symmetry to every individual atom in the system, we have made use of a particular quantification of the angular environment of each atom $i$. This was inspired by work of Steinhardt et al. ${ }^{15}$ First, six numbers $Q_{i}^{(2 \lambda)}$, with $\lambda=1,2, \ldots, 6$, are calculated for atom $i$,

$$
Q_{i}^{(2 \lambda)}=\frac{1}{Z_{i}} \sqrt{\sum_{k}^{Z_{i}} \sum_{j}^{Z_{i}} P^{(2 \lambda)}\left(\cos \theta_{k i j}\right)}, \quad \lambda=1,2, \ldots, 6,
$$

where $P^{(2 \lambda)}$ is the Legendre polynomial of degree $2 \lambda, \theta_{k i j}$ is the triplet angle subtended at atom $i$ formed by the vectors from $i$ to its neighboring atoms $k$ and $j$, and $Z_{i}$ is the coor-
TABLE I. Numbers $Q^{(2 \lambda)}$ for an atom in different angular environments: fcc, hcp, bcc, diamond, and icosahedral. $Z$ is the coordination number of the atom. In colored figures, the local symmetry types are colored as indicated; an unclassified symmetry type appears as magenta.

\begin{tabular}{clllll}
\hline \hline & \multicolumn{5}{c}{$Q^{(2 \lambda)}$} \\
\cline { 2 - 6 } $2 \lambda$ & fcc & hcp & bcc & Diamond & Icosohedral \\
\hline 2 & 0 & 0 & 0 & 0 & 0 \\
4 & 0.191 & 0.097 & 0.036 & 0.509 & 0 \\
6 & 0.575 & 0.485 & 0.511 & 0.629 & 0.663 \\
8 & 0.404 & 0.317 & 0.429 & 0.213 & 0 \\
10 & 0.013 & 0.010 & 0.195 & 0.650 & 0.363 \\
12 & 0.600 & 0.565 & 0.405 & 0.415 & 0.585 \\
& & & & & 12 \\
$Z$ & 12 & 12 & 14 & 4 & Cyan \\
Color & Green & Orange & Blue & Yellow & \\
\hline \hline
\end{tabular}

dination number of $i$. Note that the lengths of the vectors play no role other than selecting or excluding the atoms $k$ and $j$ as nearest neighbors. The maximum nearest-neighbor distances were chosen as $3.27 \AA$ for $\mathrm{Cu}-\mathrm{Cu}, 3.46 \AA$ for $\mathrm{Cu}$ Ta, and $3.66 \AA$ for Ta-Ta. For atoms in perfect fcc, hcp, bcc, diamond cubic, and icosahedral environments, the values of $Q^{(2 \lambda)}$ are shown in Table I. These five structures are the candidate structures used in this work. To every atom $i$ in the system under study one of these is assigned as the local symmetry type, provided that the actual environment of the atom and the environment in the assigned candidate structure are not too different. For atom $i$ we therefore calculate the rms differences of the first six even orders $Q_{i}^{(2 \lambda)}$ with the corresponding $Q_{\mathrm{struc}}^{(2 \lambda)}$ of each of these candidate structures,

$$
\Delta S_{i, \text { struc }}=\sqrt{\frac{1}{6} \sum_{\lambda=1}^{6}\left(Q_{i}^{(2 \lambda)}-Q_{\text {struc }}^{(2 \lambda)}\right)^{2}} .
$$

The structure for which $\Delta S$ has the smallest value is attributed to atom $i$ as its local symmetry type, provided that this $\Delta S$ is smaller than 0.11 . This critical bound was determined by numerical experimentation. If $\Delta S$ is greater, the local crystal symmetry of $i$ is recorded as "unclassified." In this way we can divide up the full system into fcc, hcp, bcc regions, etc., which after visualization are useful for detecting different crystal defects, as we will see. Note that local symmetry type is an atomic property derived from the neighbors of the atom in three dimensions, not just from the neighbors that lie in the same plane of deposition. In this work we will show that the local symmetry type is a very useful tool for clarifying the microstructure of the film, although the three-dimensional (3D) character of the local symmetry type calls for cautious interpretation of in-plane domains in some cases.

\section{RESULTS: THE CU FILM AFTER DEPOSITION}

Before presenting the results of the deposition simulations, we note that all results in this work are shown for $0 \mathrm{~K}$ 
TABLE II. Data of the atomic planes after film deposition, top to bottom (see also Figs. 1 and 2). $N_{\text {Ta }}$, $N_{\mathrm{Cu}}$, and $N_{\text {tot }}$ are the actual numbers of atoms in the plane; $n_{\mathrm{Cu}}$ is the hypothetical number of $\mathrm{Cu}$ atoms in the plane if the areas outside the terrace area would be representative for the whole plane.

\begin{tabular}{|c|c|c|c|c|c|c|}
\hline Plane & $N_{\mathrm{Ta}}$ & $N_{\mathrm{Cu}}$ & $N_{\text {tot }}$ & $\begin{array}{l}\text { rms thickness } \\
\text { of plane } \\
(\AA)\end{array}$ & $\begin{array}{c}\text { Distance to next } \\
\text { lower plane } \\
(\AA)\end{array}$ & $n_{\mathrm{Cu}}$ \\
\hline $\mathrm{Cu} 10$ & 0 & 60 & 60 & 0.41 & 1.97 & $\sim 0$ \\
\hline $\mathrm{Cu} 9$ & 0 & 3194 & 3194 & 0.13 & 1.95 & $\sim 0$ \\
\hline $\mathrm{Cu} 8$ & 0 & 34879 & 34879 & 0.17 & 1.90 & 7878 \\
\hline $\mathrm{Cu} 7$ & 0 & 107387 & 107387 & 0.21 & 1.93 & 68328 \\
\hline $\mathrm{Cu} 6$ & 0 & 163842 & 163842 & 0.21 & 2.00 & 155774 \\
\hline $\mathrm{Cu} 5$ & 1 & 173678 & 173679 & 0.21 & 2.01 & 173837 \\
\hline $\mathrm{Cu} 4$ & 7 & 173838 & 173845 & 0.20 & 2.03 & 173483 \\
\hline $\mathrm{Cu} 3$ & 23 & 172725 & 172748 & 0.21 & 1.91 & 174293 \\
\hline $\mathrm{Cu} 2$ & 37 & 135499 & 135536 & 0.10 & 0.94 & 170834 \\
\hline Ta terrace & 40977 & 31 & 41008 & 0.07 & 0.81 & \\
\hline $\mathrm{Cu} 1$ & 2 & 50816 & 50818 & 0.08 & 0.91 & 91809 \\
\hline Тa 0 & 91777 & 32 & 91809 & 0.09 & 1.72 & \\
\hline $\mathrm{Ta}-1$ & 91809 & 0 & 91809 & 0.06 & 1.64 & \\
\hline $\mathrm{Ta}-2$ & 91809 & 0 & 91809 & 0.05 & 1.66 & \\
\hline $\mathrm{Ta}-3$ & 91809 & 0 & 91809 & 0.04 & 1.66 & \\
\hline $\mathrm{Ta}-4$ & 91809 & 0 & 91809 & 0.04 & 1.65 & \\
\hline $\mathrm{Ta}-5$ & 91809 & 0 & 91809 & 0.03 & 1.60 & \\
\hline $\mathrm{Ta}-6$ & 91809 & 0 & 91809 & 0.02 & & \\
\hline
\end{tabular}

configurations, i.e., for configurations copied from the ongoing run and subsequently rapidly quenched to $0 \mathrm{~K}$. This was done to suppress thermal noise, which especially affects the determination of the local crystal symmetry of the atoms.

Table II and Fig. 2 summarize the $\mathrm{Cu}$-Ta system after the deposition of the film. In Figs. 2(c)-2(f) the four most interesting $\mathrm{Cu}$ planes, $\mathrm{Cu} 2-5$, are shown with the atoms colored according to local symmetry type. In Table II, $N_{\mathrm{Ta}}, N_{\mathrm{Cu}}$, and $N_{\text {tot }}$ are the observed number of atoms in the plane and $n_{\mathrm{Cu}}$ is the hypothetical number of $\mathrm{Cu}$ atoms in the plane if the area outside the terrace area would be representative for the whole plane. We will call the area outside the terrace area the outside area of a plane. These numbers $n_{\mathrm{Cu}}$ reflect, better than the observed numbers $N_{\mathrm{Cu}}$, the filling factor of the plane (i.e., the outside region) and therefore the defect content of the plane. Plane $\mathrm{Cu} 1$ is fully epitaxial on the bcc-Ta (100) plane Ta 0 . This follows not only from a visual inspection but also from the fact that $n_{\mathrm{Cu}}$ in plane $\mathrm{Cu} 1$ is equal to $N_{\mathrm{Ta}}$ in plane Ta 0 . The planes $\mathrm{Cu} 2-\mathrm{Cu} 5$ all have numbers $n_{\mathrm{Cu}}$ in the range $1.70 \times 10^{5}-1.74 \times 10^{5}$. To put these numbers in perspective, we note that in stress- and defect-free conditions fcc- $\mathrm{Cu}$ (111) planes of this size contain $1.77 \times 10^{5}$ atoms. This is a clear indication that plane $\mathrm{Cu} 2$ (outside area) has the most defects, followed by planes $\mathrm{Cu} 3-5$, which have smaller packing deficits. Below we will discuss the defects that are observed in these planes. The rapid decrease in $n_{\mathrm{Cu}}$ for planes $\mathrm{Cu}$ 6-9 has another reason: these planes were not yet fully covered when the deposition stopped. There is more information in Table II. First we note that in stress-free equilibrium conditions, fcc-Cu (111) planes are separated by a distance of $2.08 \AA$ and bcc-Ta (100) planes by $1.65 \AA$. Comparison with Table II shows that the surface relaxation in the $\mathrm{Cu}$ film has a considerable range, much more than in the $\mathrm{Ta}$ substrate. The first $\mathrm{Cu}$ layer has a distance of only $0.92 \pm 0.01 \AA$ to the underlying Ta plane. This is the fully epitaxial $\mathrm{Cu}$ layer on bcc-Ta (100). This shows that the initial $\mathrm{Cu}$ layer penetrates far into the Ta plane. The $\mathrm{Cu}-\mathrm{Ta}$ atomic neighbor distance between these two planes is $2.51 \AA$, which is surprisingly small. Finally, the rms thickness of each plane is listed in Table II for completeness. Note that this thickness does not contain a contribution from thermal atomic vibrations because these are absent. Therefore, the value of $0.02 \AA$ for the bottom Ta plane indicates a small curvature, possibly due to stresses.

\section{A. Plane by plane description}

In this section we describe the local crystallographic symmetries, the microstructures, and the defects observed in the atomic planes of the deposited $\mathrm{Cu}$ film. Figure 2 will serve as the main illustration for reference. On the scale of Fig. 2, individual atoms are not visible. In later sections we will therefore discuss some of the issues in more detail, showing atomic-scale patterns and arrangements, which justify the conclusions on the microstructure presented in this summary. For brevity we mention here only the properties of the film area outside the terrace region in each plane. For the film area inside the terrace region of a plane one should refer to the underlying plane, i.e., the plane with the next lower index; this can also be seen in Fig. 2. 

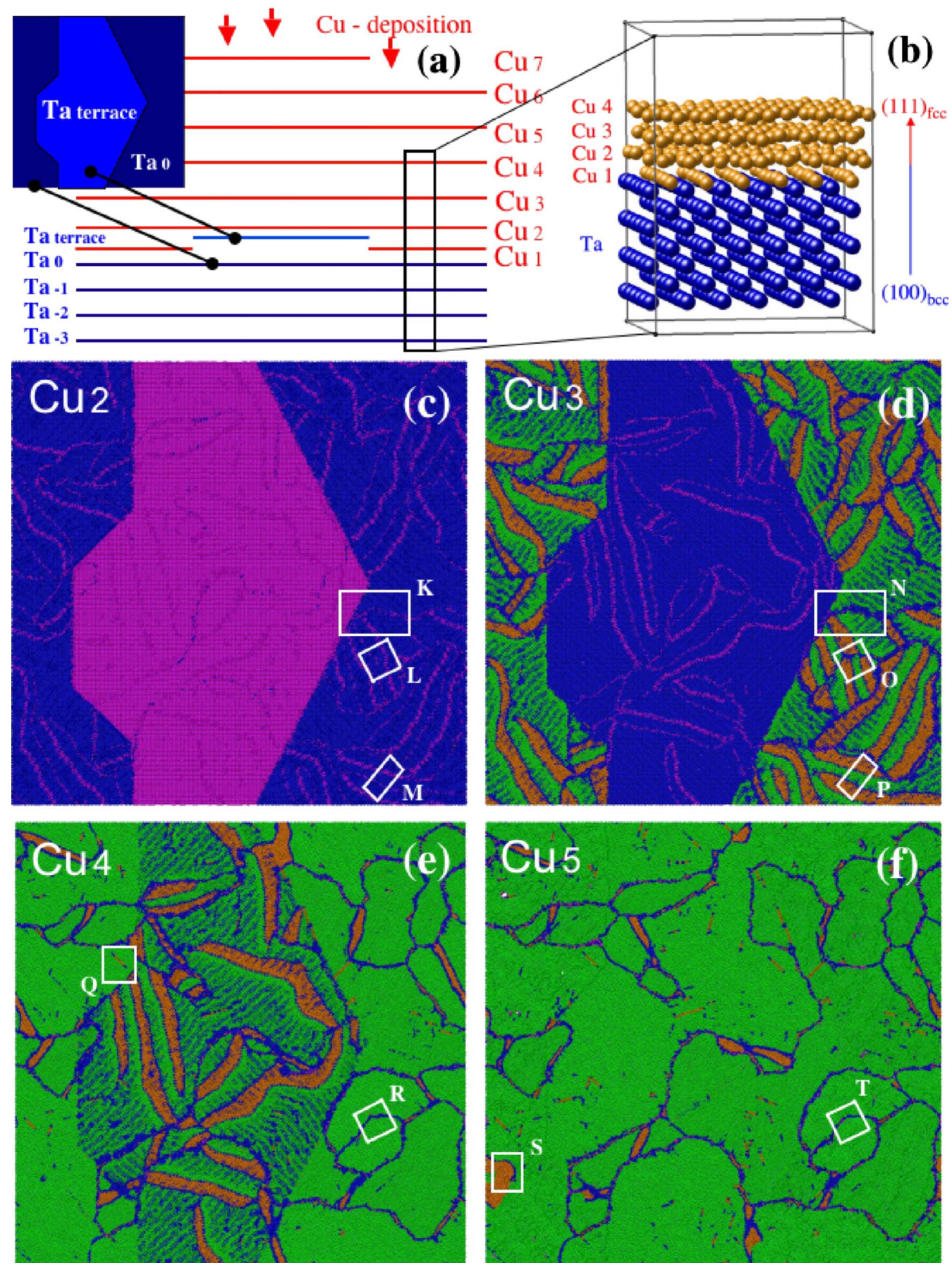

FIG. 2. (Color online) (a) Schematic side and top views as in Fig. 1(a). (b) Small section of the Cu-Ta system viewed at atomic scale (blue $=\mathrm{Ta}$, orange $=\mathrm{Cu}$ ). Note how deep the epitaxial plane $\mathrm{Cu} 1$ has sunk into the top Ta layer. (c)-(f) Top views of the planes $\mathrm{Cu} 2-5$ after deposition. The atoms are colored according to local symmetry type ( green $=\mathrm{fcc}$, blue $=\mathrm{bcc}$, orange $=\mathrm{hcp}$, magenta $=$ unclassified). Boxes denote regions discussed in the main text.

Plane $\mathrm{Cu}$ 1. Being fully epitaxial on bcc-Ta (100), plane $\mathrm{Cu} 1$ contains no defects. As mentioned above, it sinks deep into the top Ta layer. Because of this, and because the $\mathrm{Cu} 2$ layer above it is not a bcc (100) plane, the first deposited $\mathrm{Cu}$ layer is not recognized by the local symmetry algorithm as having bec symmetry type but as being unclassified. This can be seen in the terrace area in Fig. 2(c), which is the part of plane $\mathrm{Cu} 2$ that is in fact the first deposited $\mathrm{Cu}$ plane.

Plane $\mathrm{Cu}$ 2. In Fig. 2(c), plane $\mathrm{Cu} 2$ shows up as blue, but it has no true 3D bcc symmetry. As we will show later, this plane has a misfit superstructure with a $(7 \sqrt{ } 2 \times \sqrt{ } 2) R 45^{\circ}$ supercell and is in a sense intermediate between bcc (100) and fcc (111) planes. The magenta curved lines in $\mathrm{Cu} 2$ are GBs or rather the beginning of GBs which stretch out upward. There are two types of GBs:

(1) GBs between areas of different in-plane orientations (two such orientations occur, which will be designated as I and II) and 
(2) GBs between areas of identical in-plane orientation but shifted by in-plane vectors of the type $(1 / 6)\langle 211\rangle$, i.e., areas with three possible "anchor positions" A-C, as they are usually indicated in stacking sequences of close-packed planes. This terminology is used as if this plane is a genuine fcc (111) plane.

Also the relatively low number of atoms in this plane $\left(n_{\mathrm{Cu}}=170834\right)$ compared to the next higher planes (Table II) shows that there is a packing deficit in this plane.

Plane $\mathrm{Cu} 3 . \mathrm{Cu} 3$ is the first fcc (111) plane but one with many defects visible in Fig. 2(d). Since from this plane upward all planes and their neighboring planes above and below are essentially fcc (111) planes, green is the main color for these planes. GBs show up mainly as blue. At this point it should be illustrated why the local symmetry type should be interpreted with caution, and why the true defect content of plane $\mathrm{Cu} 3$ is much less than Fig. 2(d) suggests. The reason is as follows. An orange area (hcp) denotes a stacking fault plane, one for which the next lower and higher planes have the same anchor positions (such as, for example, in the vertical stacking sequence $\mathrm{A}, \mathrm{B}, \mathrm{A}$ in planes $2,3,4$ ) rather than different anchor positions (such as A,B,C in planes 2,3,4). In the first of these sequences the B plane shows up as hcp (orange); in the second the B plane shows up as fcc (green). Therefore, neighboring orange and green regions in the same plane do not necessarily have different anchor positions. Instead, the different colors may be the result of different anchor positions in the next lower or upper plane. The A,B,A, and $\mathrm{A}, \mathrm{B}, \mathrm{C}$ examples just given illustrate this: the $\mathrm{B}$ region may show up as orange or as green. The "boundary" between these two $\mathrm{B}$ regions is then artificial (nonexistent). The true GB is one plane up or down. Note that a pair of orange planes, at least if they are adjacent planes, denotes an intrinsic stacking fault, and these have indeed been found (see plane $\mathrm{Cu} 4$ ). Another notable phenomenon is the blue stripes in plane $\mathrm{Cu} 3$. We will show that they are associated with the presence of misfit supercells in the underlying plane $\mathrm{Cu} 2$. Stripes run in the direction normal to the orientation (I or II) of the grain in plane $\mathrm{Cu} 2$. Later we will show that the occurrence of these stripes and the fact that the orange areas all have approximately the same width are intimately connected with misfit supercells formed in plane $\mathrm{Cu} 2$.

Plane $\mathrm{Cu}$ 4. As seen in Fig. 2(e), plane $\mathrm{Cu} 4$ is also an imperfect fcc (111) plane, but less imperfect than the underlying plane. There are no more stripes, which indicates that the supercell modulations in plane $\mathrm{Cu} 2$ do not propagate upward upon growth. GBs are still present in two modifications: between different orientations (I and II) and between different anchor positions $(\mathrm{A}-\mathrm{C})$. An example of the second one is the boundary between the two regions in box R. Close inspection shows that the regions have the same orientation, but that - although they both are green-they are $(1 / 6)\langle 211\rangle$ vector shifted. The blue boundaries in the plane below, in box $\mathrm{O}$ in Fig. 2(d), are actually artificial according to the explanation given for plane $\mathrm{Cu} 3$. To fully understand the nature of the boundary in box R, one should turn to Table III and follow the vertically stacked regions in the boxes $\mathrm{L}, \mathrm{O}$, $\mathrm{R}$, and T, which appear in Figs. 2(c)-2(f), respectively. The orange lines bounded by two blue dots, such as seen in, e.g., box $\mathrm{Q}$ in Fig. 2(e), are intrinsic stacking faults, bounded by two Shockley partial dislocations, of which the stacking fault strip runs approximately vertically.

Plane $\mathrm{Cu}$ 5. This is the first plane that looks homogeneous, in the sense that the terrace and outside areas look very similar [see Fig. 2(f)]. There are just a few "imprints" of the original step edge visible in this plane, but they are not very obvious. We conclude that the influence of the Ta terrace on the film microstructure is minimal. As the current step edges run in three distinct low-index directions, it is not expected that higher-index directions-being in a sense interpolations of the low-index directions-would suddenly have a pronounced influence. The GBs run vertically. Compared to plane 4, more Shockley partials are visible here. Some 65 intrinsic stacking faults were identified in the whole system, eight of them very near the original step-edge locations on the Ta substrate and the rest distributed within the grains. Also one growth stacking fault is observed [box $\mathrm{S}$ in Fig. 2(f)]. This stacking fault is the usual one, in the sense that the Ta lattice did not influence its formation and shape. Note that it is not a "long orange patch" like those seen in plane $\mathrm{Cu} 3$.

Plane $\mathrm{Cu} 6$ and higher. Upward from plane 5, the planes are no longer fully occupied [see also Fig. 1(b)]. The microstructure of the underlying planes is continued, however. As the coverage decreases, i.e., in increasingly higher planes, the formation of islands is clear. To give a numerical illustration: planes 7 (outside) and 8 (terrace area) are approximately $40 \%$ occupied and consist of irregular islands with a mean size of $60 \AA$.

Before moving on to the atomic-scale description of the defects, we should return once more to plane $\mathrm{Cu} 4$ [Fig. 2(e)] and compare the microstructure of its outside area with that shown in Fig. 3. Also in Fig. 3 a $\mathrm{Cu} 4$ plane is depicted, but here the result was taken from the simulation on the much smaller system mentioned earlier $\left(18 \times 18 \mathrm{~nm}^{2}\right) .{ }^{1}$ In the smaller system several intrinsic stacking faults are visible but not any of the other phenomena seen in Fig. 2(e), like growth stacking faults or grain boundaries. This comparison clearly shows how simulations on too small systems may hide essential features of the physics under study. It is one of the key motivations of presenting these results.

\section{B. Atomic-scale descriptions}

In this section we illustrate in atomic detail some of the features that were observed in the film: grain orientations and grain boundaries, misfit supercells, and the origin of the narrow orange strips in plane $\mathrm{Cu} 3$ [Fig. 2(d)]. As far as point defects are concerned, almost no vacancies were found: only several tens in a system of over a million deposited atoms.

Grain orientations and grain boundaries. Figure 4 shows an atomic view of box $\mathrm{K}$ in Fig. 2(c) and is therefore a close up of plane $\mathrm{Cu} 2$. Figure 4 summarizes the different $\mathrm{Cu}$ grains that are found in the film. The coloring of the atoms is by local symmetry type, just as in Fig. 2(c). On the left of Fig. 4 is the bcc (100) part of this $\mathrm{Cu}$ plane, which is epitaxial on the Ta terrace just below it. Across the GB indicated by the blue dashed line, on the right of it, one sees the typical atomic arrangement of the outside area of plane $\mathrm{Cu} 2$. As 
TABLE III. (Color online) Schematic illustration of boundaries between grains having the same in-plane orientation but having different anchor positions A, B, or C. Areas inside the rectangles below represent the areas of boxes $\mathrm{L}, \mathrm{O}, \mathrm{R}$, and $\mathrm{T}$ in Fig. 2, elongated and rotated counterclockwise by $\sim 60^{\circ}$. Solid lines represent grain boundaries (possibly artificial ones); at the position of dashed lines there may be boundaries in other planes. As an example of how the color of a grain is determined, we look at box O. All grains in box $\mathrm{O}$ have anchor point $\mathrm{B}$. If similar areas in boxes $\mathrm{R}, \mathrm{O}$, and $\mathrm{L}$ have three different anchor point positions, then the crystal structure of that area in box $\mathrm{O}$ will be determined as fcc (green). If the positions in boxes $\mathrm{R}$ and $\mathrm{L}$ are the same for part of the area of the box, then that part will be determined to have hcp structure (yellow). Once the colors of all parts of the boxes are know, the nature of the boundaries can be determined. The text next to each box explains the type of boundaries found in each box.

\begin{tabular}{|c|c|c|c|c|}
\hline Plane & \multicolumn{3}{|c|}{$\begin{array}{l}\text { Selected area in Figs. 2(c)-2(f) } \\
\text { schematically shown }\end{array}$} & Explanation \\
\hline $\mathrm{Cu} 6$ & & $\mathrm{~B}$ & B. & $\begin{array}{l}\text { Boundaries are no longer present. The whole area } \\
\text { is } \mathrm{B} \text {, just as the area below in box } \mathrm{O} \text {. To maintain } \\
\text { the fcc structure a split of the regions is no longer } \\
\text { required. Plane } \mathrm{Cu} 6 \text { is not shown in Fig. } 2 \text {. }\end{array}$ \\
\hline $\mathrm{Cu} 5$ & $\begin{array}{c}\text { Box T } \\
\text { Fig. 2(f) }\end{array}$ & C & A & $\begin{array}{l}\text { The blue line is the boundary between different } \\
\text { anchor position areas } \mathrm{C} \text { and A. The split of the } \\
\text { regions is necessary to maintain the fcc structure } \\
\text { within both of them. }\end{array}$ \\
\hline $\mathrm{Cu} 4$ & $\begin{array}{l}\text { Box R } \\
\text { Fig. 2(e) }\end{array}$ & 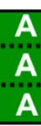 & C... & $\begin{array}{l}\text { The blue line is the boundary between different } \\
\text { anchor position areas A and C. Formed freely, no } \\
\text { influence of the Ta substrate. }\end{array}$ \\
\hline $\mathrm{Cu} 3$ & $\begin{array}{l}\text { Box O } \\
\text { Fig. 2(d) }\end{array}$ & $\begin{array}{l}\mathbf{B} \\
\mathbf{B} \\
\mathbf{B}\end{array}$ & $\begin{array}{l}\mathrm{B} \\
\mathrm{B} \\
\mathrm{B}\end{array}$ & $\begin{array}{l}\text { All blue boundaries are artificial, indicating region } \\
\text { shifts from } \mathrm{A} \text { to } \mathrm{C} \text { in plane } \mathrm{Cu} 2 \text { (horizontal lines) } \\
\text { and in plane } \mathrm{Cu} 4 \text { (vertical line). The whole area } \\
\text { is close-packed } \mathrm{B} \text { (111) fcc or hcp. }\end{array}$ \\
\hline $\mathrm{Cu} 2$ & $\begin{array}{l}\text { Box L } \\
\text { Fig. 2(c) }\end{array}$ & $\begin{array}{l}\text { C } \\
\text { A } \\
\text { C }\end{array}$ & $\begin{array}{l}\text { C } \\
\text { A } \\
\text { C }\end{array}$ & $\begin{array}{l}\text { The magenta lines are GBs between different } \\
\text { anchor position areas A and C. Formed under the } \\
\text { influence of the Ta bcc substrate below (see the } \\
\text { main text for an explanation). }\end{array}$ \\
\hline
\end{tabular}

said earlier, it is intermediate between bcc (100) and fcc (111) planes, and we will describe it in the terminology of an fcc (111) plane. The two groups of three parallel lines shown in Fig. 4 run in the two different close-packed fcc $\langle 110\rangle$ directions that are found throughout the copper film; the associated atomic hexagons are also shown in the figure. These two fcc $\langle 110\rangle$ directions make angles of $+45^{\circ}$ or $-45^{\circ}$ with the bcc [100] direction of the Ta substrate, and they are designated as orientations I and II, respectively. In the figure, four grains are visible: two having anchor position A with different grain orientations (I and II) and two having anchor position $\mathrm{C}$ with the same grain orientation (II). The $\mathrm{A}_{\mathrm{II}}$ and $\mathrm{C}_{\mathrm{II}}$ grains have the same grain orientation but they are translated by $(1 / 6)\langle 211\rangle$ vectors. For A-B and B-C grain combinations the same translation vectors apply. The red dashed lines in the figure indicate grain boundaries of various types: boundaries between orientation differences $\left(\mathrm{A}_{\mathrm{I}}\right.$ and $\left.\mathrm{A}_{\mathrm{II}}\right)$, between anchor position differences $\left(\mathrm{A}_{\mathrm{II}}\right.$ and $\left.\mathrm{C}_{\mathrm{II}}\right)$, and between both of these $\left(\mathrm{A}_{\mathrm{I}}\right.$ and $\left.\mathrm{C}_{\mathrm{II}}\right)$.

It is seen that in this, plane $\mathrm{Cu} 2$, the local symmetry of many atoms in these GBs is colored magenta. Following these types of GBs through Figs. 2(c)-2(f) one notices that

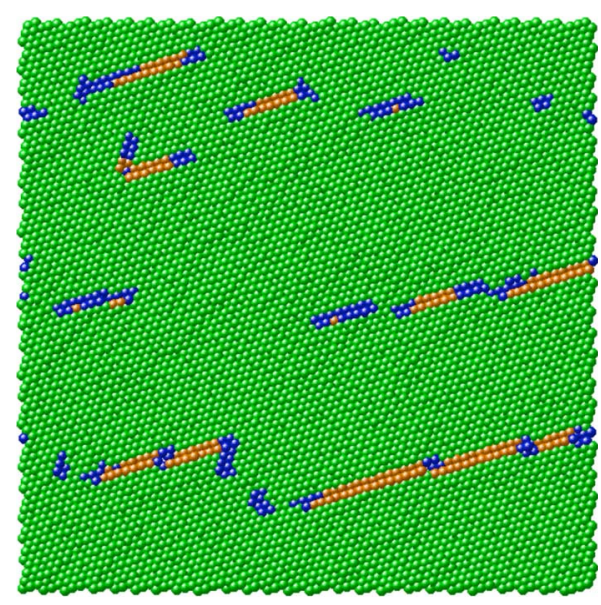

FIG. 3. (Color online) Plane $\mathrm{Cu} 4$ from a $\mathrm{Cu}$ deposition on an $18 \times 18 \mathrm{~nm}^{2}$ flat bcc-Ta (100) substrate (Ref. 1). Note the differences with the outside area of plane $\mathrm{Cu} 4$ of Fig. 2(e), which was produced under the same deposition conditions but on a 100 $\times 100 \mathrm{~nm}^{2}$ substrate. Clearly the boundary conditions of the small substrate have suppressed defects such as grain boundaries. 


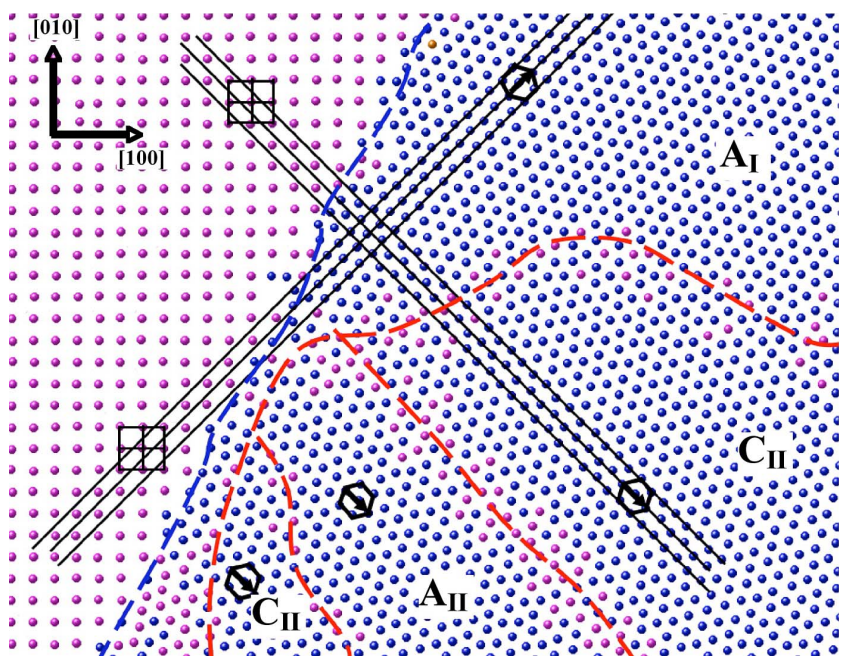

FIG. 4. (Color online) Atomic-level view of box K in Fig. 2(c). The atoms are all in plane $\mathrm{Cu} 2$ and are colored by local symmetry type. On the left is the bcc (100) part of this $\mathrm{Cu}$ plane, which is epitaxial on the Ta terrace just below it. Across the blue dashed grain boundary, on the right of it, is the typical atomic arrangement of the outside area of plane $\mathrm{Cu} 2$. Described in the terminology of an fcc (111) plane, the two groups of three parallel lines run in the two different close-packed $\langle 110\rangle$ directions; the associated atomic hexagons are also shown. These two fcc $\langle 110\rangle$ directions make angles of $+45^{\circ}$ or $-45^{\circ}$ with the bcc [100] direction of the Ta substrate, and they are designated as orientations I and II, respectively. In the figure, four grains are visible: two having anchor position $\mathrm{A}$ and two having anchor position $\mathrm{C}$. The $A_{\mathrm{II}}$ and $C_{\mathrm{I}}$ grains are translated by $(1 / 6)\langle 211\rangle$ vectors, and so are-although not shown here $-A_{\mathrm{I}}$ and $C_{\mathrm{I}}$ grains. For A-B and B-C grain combinations the same translation vectors apply. The red dashed lines in the figure indicate grain boundaries of various types: boundaries between orientation differences $\left(A_{\mathrm{I}}\right.$ and $\left.A_{\mathrm{II}}\right)$, between anchor position differences $\left(A_{\mathrm{II}}\right.$ and $\left.C_{\mathrm{II}}\right)$, and between both of these $\left(A_{\mathrm{I}}\right.$ and $\left.C_{\mathrm{II}}\right)$.

many of the GBs between grains with different orientations run upward (and show up as blue in planes $\mathrm{Cu} 3$ and higher). These are $30^{\circ}[111]$ fcc tilt grain boundaries. However, as explained earlier, the GBs between grains with different anchor points in plane $\mathrm{Cu} 2$ give rise to orange patches in plane $\mathrm{Cu} 3$; see, for example, box $\mathrm{N}$ in Fig. 2(d) and compare with Fig. 4 [magnified box $\mathrm{K}$ in Fig. 2(c)]. Above plane 3, Figs. 2(e) and 2(f) show that these GBs between grains with different anchor points have largely disappeared. However, they are still present, and Table III explains their propagation. Only in plane 3 these GBs are completely absent. The orange patches and their blue boundaries say nothing about the anchor positions of the grains in plane $\mathrm{Cu} 3$ but only reflect the difference in anchor positions of the underlying grains in plane $\mathrm{Cu} 2$. The only real boundaries in plane $\mathrm{Cu} 3$ are those between grains of different orientations. In addition to the orange patches, plane $\mathrm{Cu} 3$ exhibits one more special feature: blue stripes [see Fig. 2(d)]. We will discuss the orange patches and the blue stripes next, because they are both related to the misfit supercells in plane $\mathrm{Cu} 2$.

Misfit supercells. One of the most interesting results of the local symmetry analysis is the observation of blue stripes in the third deposited $\mathrm{Cu}$ layer. They are not tiny strips of bcc
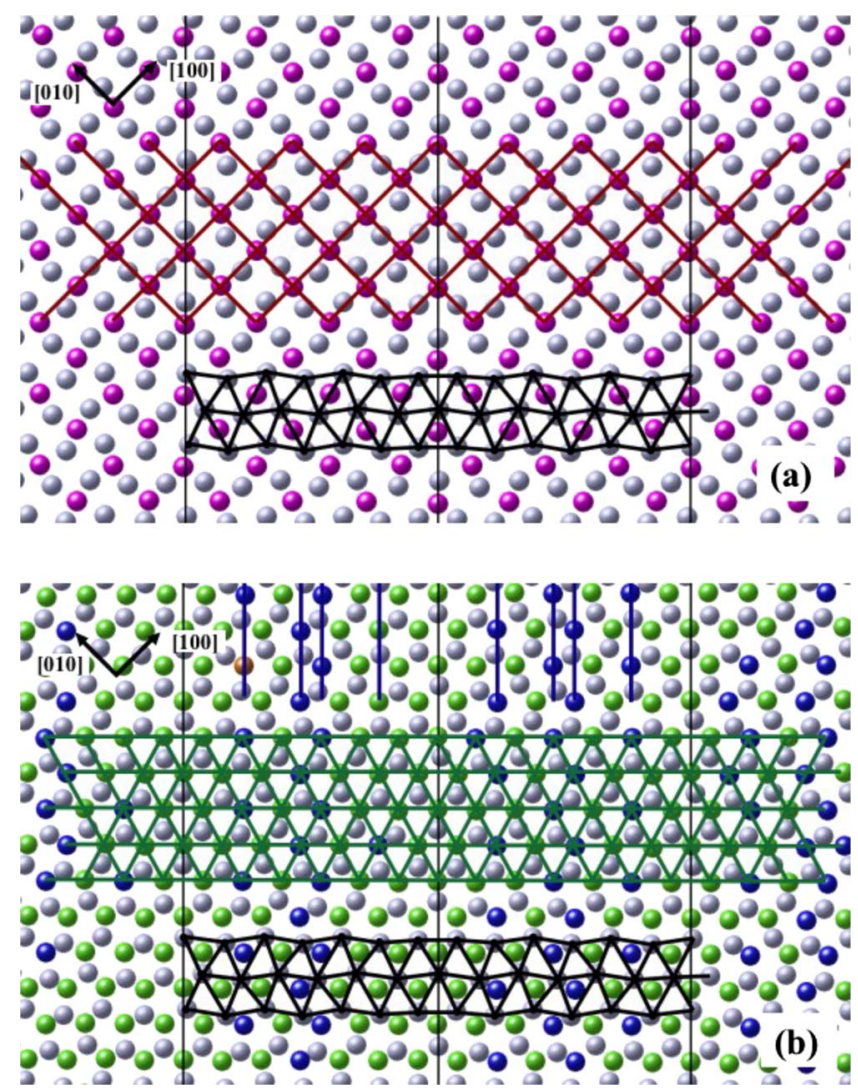

FIG. 5. (Color online) Illustration of the 26-atom misfit supercell in plane $\mathrm{Cu} 2$ (gray atoms), shown connected by black triangles. The area shown is a close-up of the $C_{\mathrm{II}}$ grain in Fig. 4, but rotated over $45^{\circ}$. In (a), plane $\mathrm{Cu} 2$ is shown together with the underlying plane $\mathrm{Cu} 1$ [magenta atoms, connected by bcc (001) squares]. In (b), plane $\mathrm{Cu} 2$ is shown together with the plane above it, $\mathrm{Cu} 3$ [green and blue atoms, connected by fcc (111) triangles]. The blue lines are shown to emphasize the atoms in the "blue stripes." Colors, except gray, represent local symmetry.

phase, as the color would suggest, but they are the result of the misfit supercells formed in the second deposited $\mathrm{Cu}$ layer, which happen to generate arrays of blue atoms in the symmetry analysis of the next higher layer. This could be concluded from an atomic-level analysis of the $\mathrm{Cu} 2$ plane. Figure 5 shows in detail [magnified box $\mathrm{K}$ in Fig. 2(c)] how plane $\mathrm{Cu} 2$ is intermediate between plane $\mathrm{Cu} 1$, a bcc (100) plane with the Ta lattice constant, and plane $\mathrm{Cu} 3$ [magnified box $\mathrm{N}$ in Fig. 2(d)], an fcc (111) plane with the $\mathrm{Cu}$ lattice constant. The figure displays part of grain $\mathrm{C}_{\mathrm{II}}$ shown in Fig. 4. Figure 5(a) shows the atoms in plane $\mathrm{Cu} 2$ (gray) together with the atoms in the next lower plane $\mathrm{Cu} 1$ (magenta), while Fig. 5(b) shows the atoms in plane $\mathrm{Cu} 2$ (gray) together with the atoms in the next higher plane $\mathrm{Cu} 3$ (green and blue). It is interesting to see how this intermediate plane $\mathrm{Cu} 2$ is built up. In both Figs. 5(a) and 5(b) the lower strip of atoms connected by black triangles is the misfit supercell in plane $\mathrm{Cu}$ 2. The supercell is a compromise between the rectangular atomic pattern in the plane below and the perfect triangular pattern of the plane above. In terms of the underlying bcc (100) $\mathrm{Cu} 1$ layer, it is a $(7 \sqrt{ } 2 \times \sqrt{2}) R 45^{\circ}$ supercell, containing $26 \mathrm{Cu}$ atoms. Figure 5(a) shows that in the [1]0] (hori- 
zontal) direction, $13 \mathrm{Cu}-\mathrm{Cu}$ nearest-neighbor bonds match the combined length of seven diagonals of the underlying $\mathrm{Cu}$ squares or, equivalently, the combined length of $7 \sqrt{ } 2$ Ta lattice constants. As 13 unstrained $\mathrm{Cu}-\mathrm{Cu}$ bonds would measure $13 \times 2.555 \AA=33.22 \AA$ for the potential used, and $7 \sqrt{ } 2 \mathrm{Ta}$ lattice constants add up to $7 \sqrt{ } 2 \times 3.303 \AA=32.70 \AA$, the supercell is under a $1.6 \%$ horizontal compressive strain with respect to the underlying plane, and this strain is accommodated by the slight zigzag in the horizontal lines connecting the $\mathrm{Cu}$ atoms in Fig. 5. In the [110] (vertical) direction, two $\mathrm{Cu}$ triangle heights, or equivalently $\sqrt{3} \mathrm{Cu}-\mathrm{Cu}$ nearest-neighbor bonds, match one diagonal of the underlying $\mathrm{Cu}$ squares or, equivalently, of $\sqrt{2}$ Ta lattice constants. In this direction the size ratio is therefore $[2 \times(\sqrt{ } 3 / 2) \times 2.555] /(\sqrt{ } 2 \times 3.303)=0.947$, and hence the supercell is under $5.3 \%$ vertical tensile strain with respect to the underlying plane. Figure 5(b) shows why the supercell in plane $\mathrm{Cu} 2$ assumes this particular $(7 \sqrt{ } 2 \times \sqrt{ } 2) R 45^{\circ}$ shape. At the cost of relatively small strains with respect to plane $\mathrm{Cu} 1$, which can be well accommodated by a zigzag distortion, at least in the horizontal direction, the fit of plane $\mathrm{Cu} 2$ with the fcc (111) $\mathrm{Cu}$ triangles in plane $\mathrm{Cu} 3$ above it is in fact very good. Apparently then, this is how the $\mathrm{Cu}$ plane manages to interpolate between two different $\mathrm{Cu}$ planes. We will come back to the vertical direction shortly.

If such supercells would fit fully periodically in the present simulation volume (they do not), and if the plane consisting of these supercells would contain no defects, the plane would contain 170502 atoms. Table II shows that the actual value of $n_{\mathrm{Cu}}$ is 170834 for plane $\mathrm{Cu} 2$. The fact that these two occupancy numbers are in excellent agreement is less trivial than it seems because it is due to a superposition of several influences. First, the value of $n_{\mathrm{Cu}}$ was derived from a scaling relation that includes some uncertainty. Also, plane $\mathrm{Cu} 2$ does contain defects, notably grain boundaries as we have seen, and therefore a smaller occupation number than 170502 should be expected. But, on the other hand, we will show that relaxation of the supercell structure has taken place, which has opened up more positions for atoms to occupy. Together then, these three effects happen to have caused an almost exact match of the two occupation numbers. The blue stripes in plane $\mathrm{Cu} 3$ are the blue atoms in Fig. 5(b), some of which are shown connected by blue lines in the top of the figure. It can be seen that they form a regular pattern (although not perfectly everywhere) perpendicular to, and in phase with, the unit cells of the supercell.

There is another observation to make. In the vertical direction, a stack of six supercells (12 $\mathrm{Cu}$ triangles), each $\sqrt{2} \times 3.303 \AA$ high in order to fit the underlying plane, measures $28.03 \AA$. However, the total height of 12 undeformed fcc (111) $\mathrm{Cu}$ triangles, as they are present in the plane above, is $12 \times(\sqrt{ } / 2) \times 2.555 \AA=26.55 \AA$. The strain energy of the six supercells with respect to the plane above is therefore considerable. Interestingly, the total height of $12+2 / 3$ undeformed fcc (111) Cu triangles is also $28.03 \AA$. It is very well possible, therefore, that the presence of the plane above, once it is rigid enough by its firm connection to the next higher planes, makes it energetically favorable for the vertical stack of supercells in plane $\mathrm{Cu} 2$ to break into pieces. The reason is the following. If each of these pieces would be six supercells

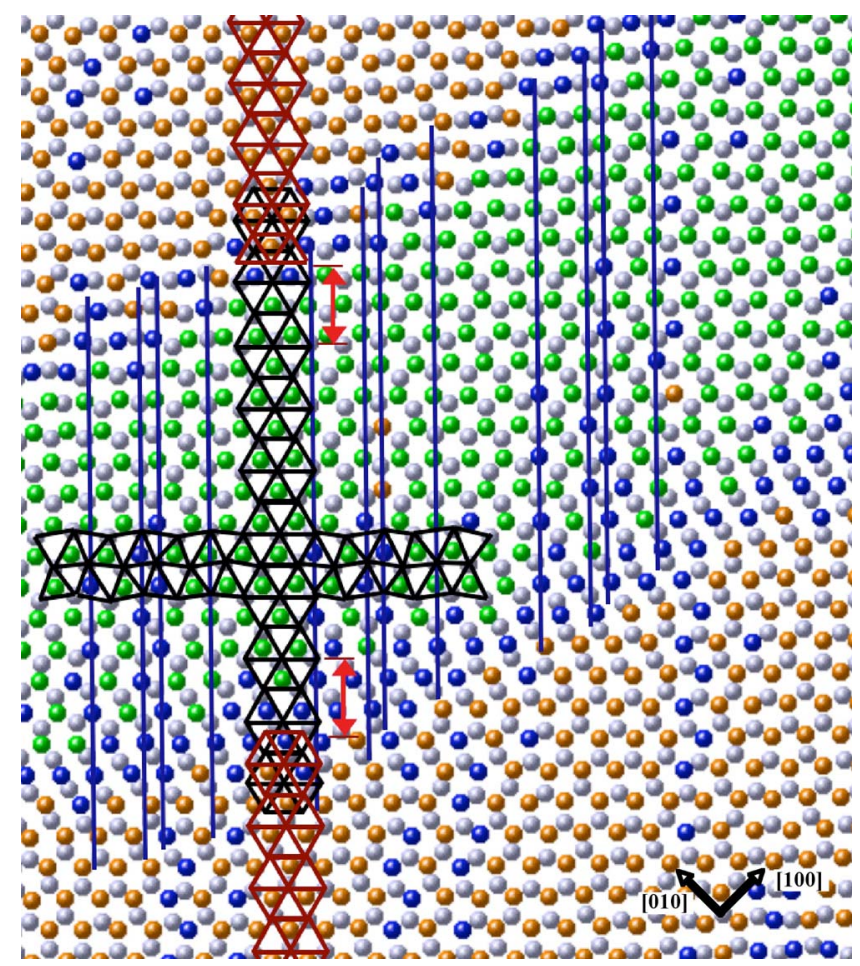

FIG. 6. (Color online) Misfit supercell, as in Fig. 5(b), only here the atomic-level view of boxes $\mathrm{M}$ (gray atoms) and $\mathrm{P}$ (green, blue, and orange atoms) in Figs. 2(c) and 2(d) is given. The central part of the supercell is shown, repeated in the vertical direction (black triangles connecting gray atoms). The red triangles also connect gray atoms, analogous to the black triangles but shifted in the vertical direction by (2/3) triangle height. This shift is due to an energetically favorable relaxation (see the main text for a discussion). Red arrows indicate the first gray atoms out of registry, near the (blue) edges of the green area. Six perfect (111) fcc hexagons fit within the green area in the vertical direction.

(12 $\mathrm{Cu}$ triangles) high, it could contract from 28.03 to $26.55 \AA$ in order to fit the plane above perfectly, while leaving the $\mathrm{Cu}$ atom that starts the next piece also in its perfect fitting position, only this perfect fitting position is shifted with respect to those of the previous piece by the $2 / 3 \mathrm{fcc}$ (111) $\mathrm{Cu}$ triangle height mentioned, or which is the same, by a $(1 / 6)\langle 211\rangle$ vector. In other words the energetic profit that plane $\mathrm{Cu} 2$ can attain to fit plane $\mathrm{Cu} 3$, once plane $\mathrm{Cu} 3$ is present and rigid enough, stimulates plane $\mathrm{Cu} 2$ to break up into pieces that are mutually shifted by $(1 / 6)\langle 211\rangle$ vectors, or-in our earlier terminology-into pieces that have different anchor positions. Figure 6 [enlarged area of boxes $M$ and $\mathrm{P}$ in Figs. 2(c) and 2(d)] confirms that this is indeed what has happened. In reality the actual number of supercells in the vertical direction is not always precisely equal to the ideal value of 6 . Nevertheless, deviations from this number are never large. This can be concluded from the fact that the widths of most orange strips in Fig. 2(d) are close to $30 \AA$. In the next section we will show that this breaking up of plane $\mathrm{Cu} 2$, or "relaxation" of plane $\mathrm{Cu} 2$ as we have called it earlier, indeed had to wait longer than the deposition of just plane $\mathrm{Cu} 3$.

The fitting together of two very different crystal structures through a single distorted atomic monolayer represents a 

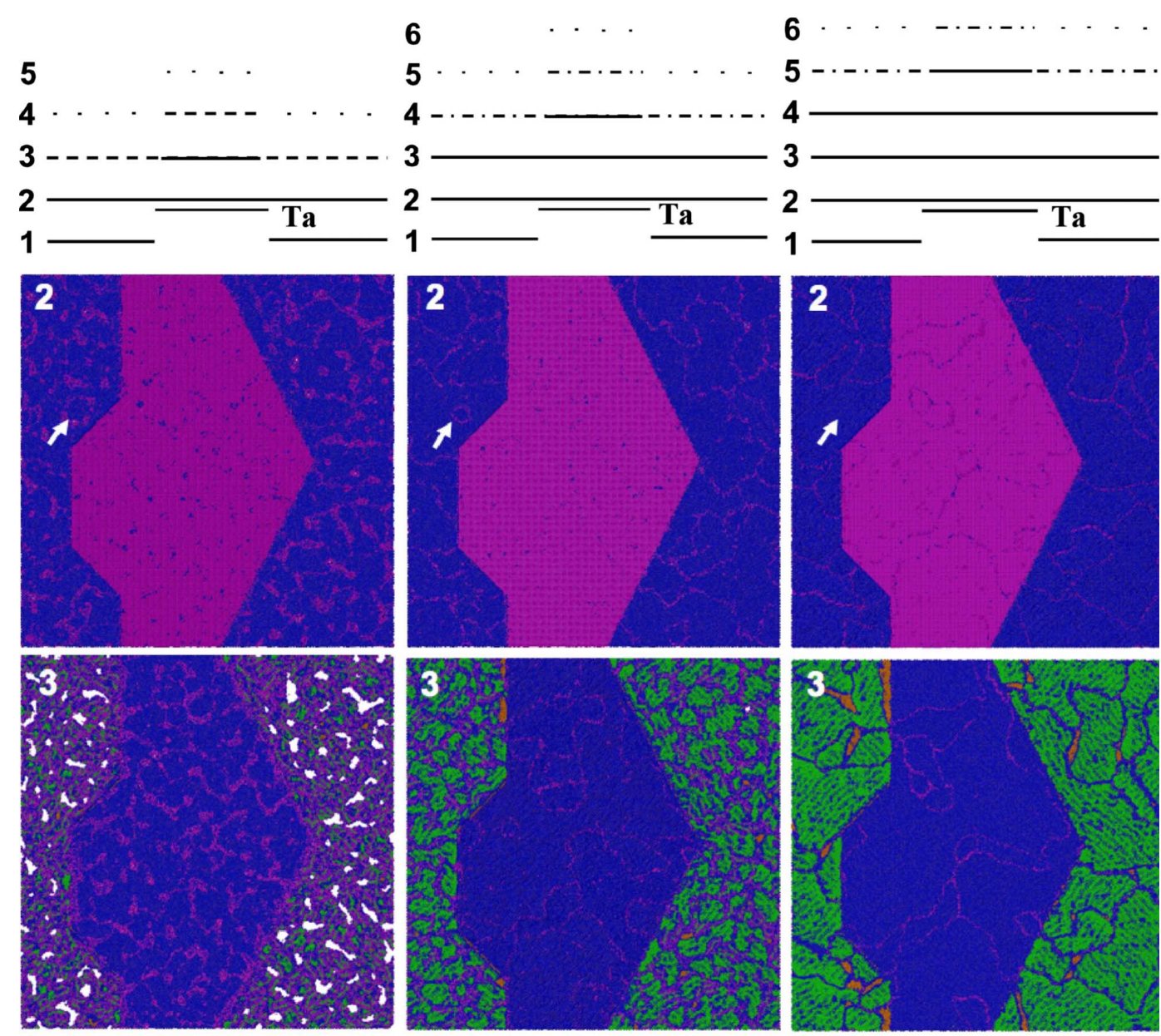

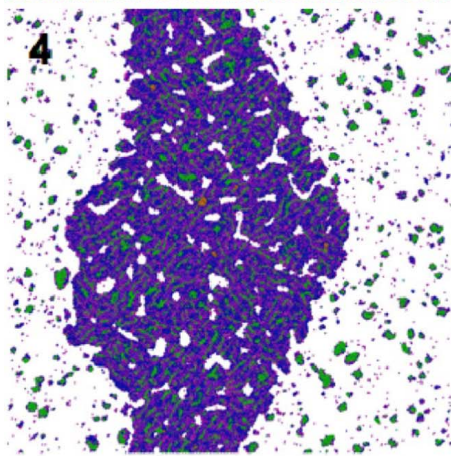

(a)

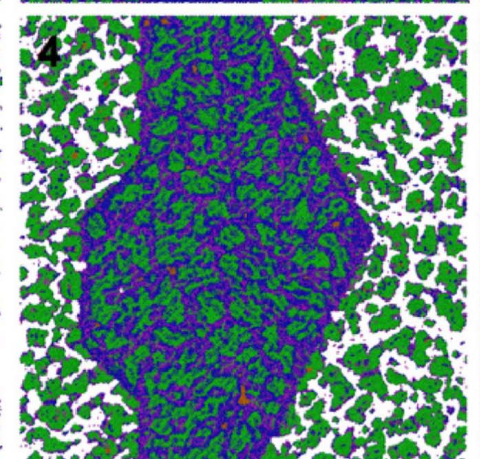

(b)

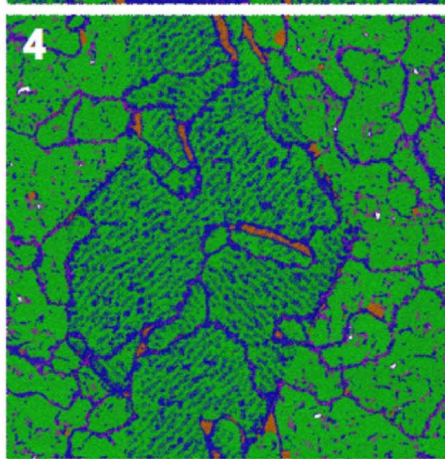

(c)

FIG. 7. (Color online) Successive stages of planes Cu 2-4 during deposition, (a) after $432091 \mathrm{Cu}$ atoms deposited or $2.96 \mathrm{ML}$, (b) after $541967 \mathrm{Cu}$ atoms deposited or 3.59 ML, (c) after $709866 \mathrm{Cu}$ deposited or 4.56 ML. Atoms are colored according to local symmetry. Plane occupancy is shown schematically at the top of the figure. The white arrows in plane $\mathrm{Cu} 2$ indicate a grain that is consumed by its surrounding grain having a different orientation. For reference we mention that the last stage of the deposition, after 6.31 ML, is shown in Fig. 2.

mechanism for enabling epitaxy that is very different from the textbook mechanism of misfit dislocations. This was also noted in recent similar studies, ${ }^{1,4}$ but in the present case the distortions and defect patterns in the critical monolayer are especially complex and revealing. This, in turn, is made possible by the free atom-by-atom growth of the atomic planes, which allows the system much greater freedom to organize itself than the joining of solid crystal slabs, a procedure with pronounced geometrical restrictions on distortions and defects. ${ }^{3,4}$

\section{CHANGES IN THE FILM DURING DEPOSITION}

Figure 7 gives an impression of the planes $\mathrm{Cu} 2-4$ in three successive stages of deposition [the fourth and final stages were already depicted in Figs. 2(c)-2(f)]. The numbers of $\mathrm{Cu}$ monolayers deposited are, respectively, 2.96, 3.59, and 4.56, and the progression of the plane occupancies is shown schematically at the top of Fig. 7. Close inspection of these results shows that in plane $\mathrm{Cu} 2$, a small grain with orientation I is consumed by the surrounding grain which has orientation 


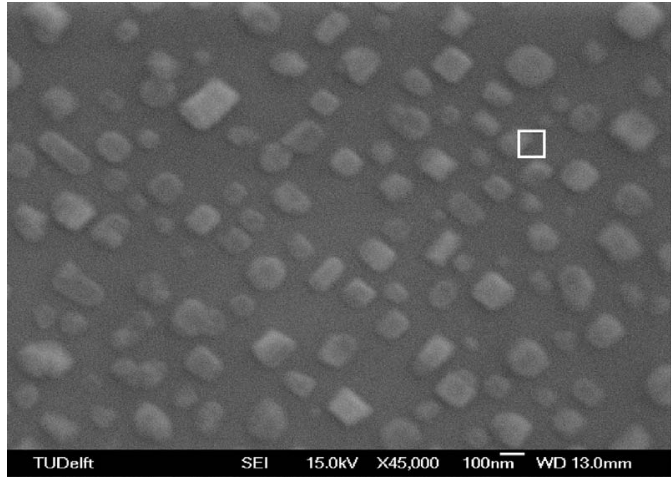

FIG. 8. Secondary electron image of a $50 \AA \mathrm{Cu}$ film deposited on Ta (100) and annealed at $995 \mathrm{~K}$ for $10 \mathrm{~s}$ (Ref. 12). The white square has the same size as the system used in the current simulations $\left(100 \times 100 \mathrm{~nm}^{2}\right)$.

II (white arrow). Also, as one follows the successive stages of plane $\mathrm{Cu} 2$ all the way to Fig. 2(c), one notices how the grain boundaries become increasingly sharper defined. One should combine this information with the changes in plane $\mathrm{Cu}$ 3, all the way to Fig. 2(d), where it is seen that the orange patches appear only in the last stage. Together this shows that the breaking up of plane $\mathrm{Cu} 2$ in regions with different anchor points, as was discussed in the previous section, is completed not before approximately $6 \mathrm{ML}$ deposition. Finally, also plane $\mathrm{Cu} 4$ in Fig. 7 is far from its final state. All this is clear evidence of the changes that occur in deposited atomic planes while the arrival of new atoms at the surface takes place several planes higher in the film.

\section{ANNEALING}

Experimentally ${ }^{14}$ it is known that $\mathrm{Cu}$ dewets bcc-Ta (100) and (110) surfaces at elevated temperatures. To be more specific, Fig. 8 shows how a 50-Å-thick copper film, deposited on a bcc-Ta (100) surface by electron gun evaporation, has dewetted the substrate after a $10 \mathrm{~s}$ anneal at $995 \mathrm{~K}$. The film breaks up and forms rectangular 3D islands, aligned along the crystallographic directions of the substrate. It could be concluded from other experiments that the process of island formation takes place from approximately 400 to $700 \mathrm{~K}$ when the $\mathrm{Cu}$-Ta system is subjected to a heating ramp of 40 $\mathrm{K} / \mathrm{s}$. The areas between the islands are not the bare, exposed Ta substrate but a $\mathrm{Cu}$ film of 1 or $2 \mathrm{ML}$ thickness remaining on top of the substrate. Hence, the experiments showed that the dewetting is actually a $\mathrm{Cu}$-on- $\mathrm{Cu}$ phenomenon, where of course the remaining $\mathrm{Cu}$ film is strained because of the underlying Ta substrate.

In order to investigate whether the $\mathrm{Cu}$ film created in the current simulations would also dewet the Ta substrate at elevated temperatures, the system was annealed after deposition of the film. For this purpose the unmodified $\mathrm{Cu}$ potentials were used, and not the $\mathrm{Cu}$ potentials modified for deposition as described earlier (Sec. II). In this way, truly elevated temperatures were simulated. It was not expected that a full island formation as shown in Fig. 8 would be found in the simulations. For this, the feasible simulation

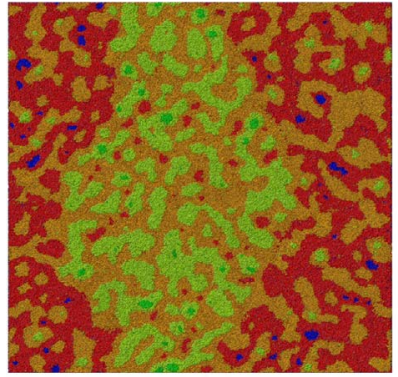

(a)

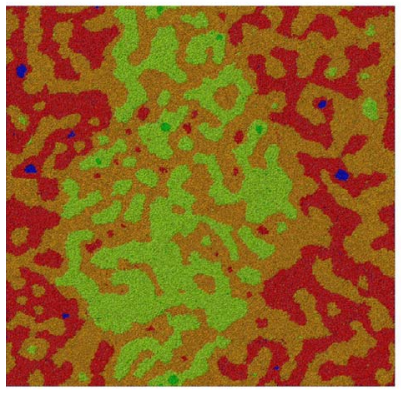

(c)

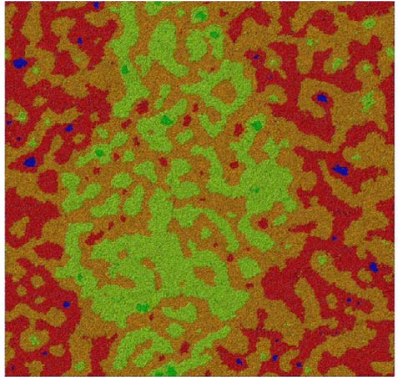

(b)

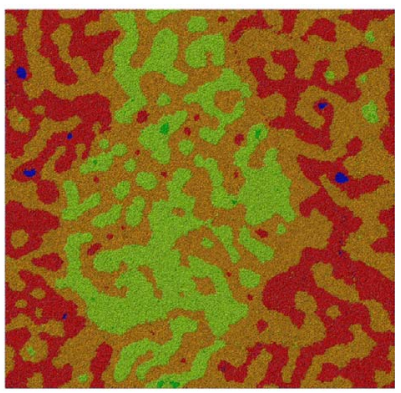

(d)
FIG. 9. (Color online) Top views of the system started from Fig. 1(b) and annealed as follows: during the first $1.77 \mathrm{~ns}$ at $550 \mathrm{~K}$, thereafter at $1000 \mathrm{~K}$. Annealing times: (a) 1.77, (b) 2.21, (c) 4.00, and (d) $5.16 \mathrm{~ns}$. The atoms are colored according to height, using a different scale than Fig. 1(b). The green atoms are in plane $\mathrm{Cu}$ 9. A coarsening of the islands is seen. For atom counts in the planes, see Table III.

times are too short, the simulation area is too small to accommodate even a single island of the size actually observed, and the $\mathrm{Cu}$ film is thinner than in the experiments. Nevertheless, it would be interesting to find out if at least a tendency toward dewetting could be identified in the simulations.

The results are shown in Fig. 9 and Table IV. First, the system was annealed at $550 \mathrm{~K}$ for $1.76 \mathrm{~ns}$ [Fig. 9(a)]. Thereafter the temperature was increased to $1000 \mathrm{~K}$ and annealing was continued for another $3.39 \mathrm{~ns}$, leading to a total annealing time of 5.16 ns [Figs. 9(b)-9(d)]. The figures clearly show that some in-plane agglomeration and coarsening of the island structure occurs. In addition, Table IV shows that the occupancy of plane $\mathrm{Cu} 7$ (the yellow atoms in Figs. 9(a)-9(d)] increases significantly, and that of the neighboring planes $\mathrm{Cu} 6$ and 8 also, but to a lesser extent. Conversely, the other $\mathrm{Cu}$ planes, from plane $\mathrm{Cu} 2$ onward, have lost atoms at the end of the annealing period. In summary then, the simulations indicate that during annealing there is horizontal as well as vertical transport of $\mathrm{Cu}$ atoms, both supporting the tendency toward agglomeration and island formation. These observations agree with what could be reasonably expected, as explained above. The much more pronounced dewetting found in the simulations by Hashibon et al. ${ }^{3}$ was the result of different simulation conditions (see Sec. I) and a higher simulation temperature $(1400 \mathrm{~K})$. This makes it almost impossible to compare the two simulation results. 
TABLE IV. Cumulative changes in the number of atoms per plane during annealing, see also [Figs. 9(a)-9(d)]. The annealing temperature during the first $1.77 \mathrm{~ns}$ was $550 \mathrm{~K}$, thereafter $1000 \mathrm{~K}$.

\begin{tabular}{|c|c|c|c|c|c|c|c|c|}
\hline \multirow[b]{2}{*}{ Plane } & \multicolumn{2}{|c|}{ After $1.77 \mathrm{~ns}$} & \multicolumn{2}{|c|}{ After $2.21 \mathrm{~ns}$} & \multicolumn{2}{|c|}{ After $4.00 \mathrm{~ns}$} & \multicolumn{2}{|c|}{ After $5.16 \mathrm{~ns}$} \\
\hline & $\Delta N_{\mathrm{Ta}}$ & $\Delta N_{\mathrm{Cu}}$ & $\Delta N_{\mathrm{Ta}}$ & $\Delta N_{\mathrm{Cu}}$ & $\Delta N_{\mathrm{Ta}}$ & $\Delta N_{\mathrm{Cu}}$ & $\Delta N_{\mathrm{Ta}}$ & $\Delta N_{\mathrm{Cu}}$ \\
\hline $\mathrm{Cu} 10$ & 0 & -15 & 0 & -18 & 0 & -20 & 0 & -18 \\
\hline $\mathrm{Cu} 9$ & 0 & -2182 & 0 & -2255 & 0 & -2564 & 0 & -2651 \\
\hline $\mathrm{Cu} 8$ & 0 & -547 & 0 & 1976 & 0 & 1741 & 0 & 1442 \\
\hline $\mathrm{Cu} 7$ & 2 & 5920 & 1 & 9572 & 1 & 11176 & 3 & 11504 \\
\hline $\mathrm{Cu} 6$ & 14 & 4687 & 21 & 3716 & 20 & 3801 & 22 & 3833 \\
\hline $\mathrm{Cu} 5$ & 9 & -1795 & 9 & -3248 & 14 & -3578 & 19 & -3579 \\
\hline $\mathrm{Cu} 4$ & 2 & -1967 & 6 & -3436 & 6 & -3785 & 5 & -3805 \\
\hline $\mathrm{Cu} 3$ & 17 & -2383 & 16 & -3952 & 34 & -4408 & 44 & -4381 \\
\hline $\mathrm{Ta} \mathrm{t}+\mathrm{Cu} 2$ & 274 & -1746 & -4 & -2418 & 35 & -2549 & 41 & -2480 \\
\hline $\mathrm{Ta} 0+\mathrm{Cu} 1$ & -316 & -11 & -47 & 18 & -109 & 122 & -138 & 73 \\
\hline $\mathrm{Ta}-1$ & -17 & 15 & 8 & 16 & 16 & 28 & 7 & 23 \\
\hline $\mathrm{Ta}-2$ & -98 & 2 & -123 & 7 & -130 & 14 & -116 & 17 \\
\hline
\end{tabular}

\section{CONCLUSIONS}

We have performed large-scale molecular-dynamics simulations using Johnson-Oh EAM potentials to study the growth and subsequent annealing of a $\mathrm{Cu}$ film (6.3 ML thickness) on a bcc-Ta (100) substrate (area of $100 \times 100 \mathrm{~nm}^{2}$ ). The main conclusions are as follows.

Compared to an earlier simulation on a considerably smaller substrate area $\left(18 \times 18 \mathrm{~nm}^{2}\right)$, a much richer microstructure is found in the current simulations. Features found here include the presence of grain boundaries throughout the simulation, misfit supercells that enable epitaxy without misfit dislocations, the splitting up of the misfit supercells as the result of continued deposition, and the coarsening and agglomeration of the surface morphology. The present results therefore clearly warn against the use of too small simulation domains, especially in cases where complex strain fields are expected, which may be too strongly limited by the boundary conditions.

The first deposited $\mathrm{Cu}$ plane grows heteroepitaxially and essentially defect free on the bcc-Ta (100) surface. The second $\mathrm{Cu}$ plane is the most interesting of all planes. It grows in the form of a superstructure of 26-atom misfit supercells which after prolonged film deposition relax energetically, by breaking up in groups and forming $30-\AA$-wide in-plane island strips separated by fcc $(1 / 6)\langle 211\rangle$ vectors. This prolonged deposition is needed to give the planes above the second plane sufficient mechanical rigidity to cause the relaxation to happen. The distorted second $\mathrm{Cu}$ monolayer represents an alternative way of enabling epitaxy between very different crystal structures without introducing misfit dislocations. The boundaries between the shifted island strips are grain boundaries of 1 or $2 \mathrm{ML}$ height. Compared to the higher planes, the second $\mathrm{Cu}$ plane contains approximately $2 \%$ fewer atoms. The third and higher $\mathrm{Cu}$ planes are fcc (111) planes, with grain boundaries as main defects. The fully deposited planes have $1.9 \%$ fewer atoms than an ideally close-packed fcc (111) plane. The $\mathrm{Cu}$ film is polycrystalline, starting from the second plane up, with two different in-plane crystal orientations. Some 65 intrinsic stacking faults bounded by Shockley partials were found. Practically no vacancies were detected. The influence of a $1 \mathrm{ML}$ Ta terrace step on the substrate is minimal. It turns out that it is not the source of interesting breaks in the film morphology. The most important (and trivial) effect is that-in the same plane-the microstructure of the part of the film that has grown on the terrace lags $1 \mathrm{ML}$ behind the microstructure of the part that has grown outside the terrace area. Upon annealing the film at elevated temperatures, a distinct tendency toward island coarsening and agglomeration is observed, but a full 3D island formation such as found experimentally is not observed.

\section{ACKNOWLEDGMENTS}

The authors would like to thank Carmen Schäfer, IMM, RWTH Aachen, Germany for discussions and critical review of the manuscript. This work is part of the research program of the Foundation for Fundamental Research on Matter (FOM), which is financially supported by the Netherlands Organisation for Scientific Research (NWO). It was carried out under Project No. 02EMM31 in the framework of the Research Program of the Materials Innovation Institute (M2i) (www.M2i.nl). 
*i.lazic@tudelft.nl

${ }^{1}$ T. P. C. Klaver and B. J. Thijsse, J. Comput.-Aided Mater. Des. 10, 61 (2003).

${ }^{2}$ T. P. Klaver and B. J. Thijsse, Magnetic and Electronic FilmsMicrostructure, Texture and Application to Data Storage, MRS Symposia Proceedings No. 721 (Materials Research Society, Pittsburgh, 2002), p. 37.

${ }^{3}$ A. Hashibon, A. Y. Lozovoi, Y. Mishin, C. Elsässer, and P. Gumbsch, Phys. Rev. B 77, 094131 (2008).

${ }^{4}$ M. J. Demkowicz and R. G. Hoagland, J. Nucl. Mater. 372, 45 (2008).

${ }^{5}$ G. V. Kurdjumov and G. Sachs, Z. Phys. 64, 325 (1930).

${ }^{6}$ D. J. Oh and R. A. Johnson, J. Mater. Res. 3, 471 (1988).

${ }^{7}$ R. A. Johnson and D. J. Oh, in Embedded Atom Method for Close-Packed Metals, Atomistic Simulations of Materials: Beyond Pair Potentials, edited by Vaclav Vitec and David J. Srolovitz (Plenum, New York, 1989), p. 233.
${ }^{8}$ R. A. Johnson and D. J. Oh, J. Mater. Res. 4, 1195 (1989).

${ }^{9}$ R. A. Johnson, Phys. Rev. B 41, 9717 (1990).

${ }^{10}$ F. R. de Boer, R. Boom, W. C. M. Mattens, A. R. Miedema, and A. K. Nienssen, in Cohesion in Metals, edited by F. R. de Boer and D. Pettifor (North Holland Physics Publishing, Amsterdam, 1989), p. 544.

${ }^{11}$ T. P. C. Klaver and B. J. Thijsse, Thin Solid Films 413, 110 (2002).

${ }^{12}$ P. T. Moseley and C. J. Seabrook, Acta Crystallogr., Sect. B: Struct. Crystallogr. Cryst. Chem. B29, 1170 (1973).

${ }^{13}$ P. Klaver, Ph.D. thesis, Delft University of Technology, The Netherlands, 2004.

${ }^{14}$ V. Venugopal and B. J. Thijsse, Thin Solid Films 517, 5482 (2009).

${ }^{15}$ P. J. Steinhardt, D. R. Nelson, and M. Ronchetti, Phys. Rev. B 28, 784 (1983). 